\title{
Distribution and morphometrical characterization of Xiphinema pachtaicum, $X$. simile and $X$. brevicollum from Hungary
}

\author{
V. REPASI ${ }^{1}$, A. AGOSTINELLI ${ }^{2}$, P. NAGY ${ }^{1 *}$, M. I. COIRO ${ }^{2}$, K. HECKER $^{1}$, F. LAMBERTI ${ }^{2 \dagger}$
}

\begin{abstract}
${ }^{1}$ Szent István University, Department of Zoology and Ecology, Gödöllö, Hungary, ${ }^{* E-m a i l: ~ n a g y . p e t e r @ m k k . s z i e . h u, ~}$ fax number: +36-28-410-804; ${ }^{2}$ Istituto per la Protezione delle Piante, Sezione di Bari, C.N.R., Via G. Amendola 168/5, 70126 Bari, Italy
\end{abstract}

\begin{abstract}
Summary
The distribution of species of the Xiphinema americanumgroup in Hungary was studied by collecting 272 samples from 53 localities. Samples have been taken from soil in the rhizosphere of 70 plant species. In total, $12.86 \%$ of the samples contained at least one species from the Xiphinema americanum-group. Three species were found: Xiphinema brevicollum, $X$. pachtaicum and $X$. simile. Xiphinema brevicollum occurred in $4.41 \%, X$. pachtaicum in $3.67 \%$ and $X$ simile in $4.77 \%$ of the samples. New data on plants associated with the reported species and developmental patterns for $X$. brevicollum and $X$. pachtaicum are also presented.
\end{abstract}

Keywords: distribution; host-range; Hungary; morphometrics; Xiphinema americanum-group

\section{Introduction}

Xiphinema is among the most important genera of plant nematodes causing direct and indirect damage to a wide variety of crops and ornamental plants worldwide. The number of species in the genus is well over 200 (Loof \& Luc, 1990) and this number is expected to grow as research continues, completed with novel methods, such as molecular taxonomy (Molinari et al., 1997, 2004; De Giorgi et al., 1999). In Central and Eastern Europe, Xiphinema has been surveyed intensely only in some countries e.g. Serbia - Barsi (1994, 1996), Slovakia - Liskova (1992), Bulgaria - Choleva (1975) and Republics of the former Soviet Union located in Europe - Brown et al. (1990). In Hungary most of the published records are over 20 years old (Andrássy, 1979; Elekes \& Valyi, 1980; Jenser, 1985). In 1990, Andrássy published a list of nematode species recorded from Hungary and, in 1999, Nagy reported the occurrence of $X$. italiae Meyl, 1953 and Longidorus attenuatus Hooper, 1961. Xiphinema brevicollum (Lordello \&
Da Costa, 1961) Luc, Coomans, Loof \& Baujard, 1998, reported as $X$. brevicolle, was found associated with raspberry (Rubus idaeus) and sour cherry (Prunus avium) (Andrássy, 1979, Jenser, 1985) while $X$. pachtaicum (Tulaganov, 1938) Kirjanova, 1951 was first mentioned to occur in Hungary, under its previous name $X$. mediterraneum Martelli \& Lamberti, 1967, associated with raspberry (Andrássy, 1979). Later, Elekes and Valyi (1980) reported it from the soil of vineyards and Jenser (1985) found it associated with sour cherry, peach $(P$. persica), apricot $(P$. armeniaca) and cherry (P. cerasus). The occurrence of $X$. simile Lamberti, Choleva \& Agostinelli, 1983 in Hungary was recently reported (Repasi et al., 2006).

In order to acquire the knowledge on the distribution of Longidorus and Xiphinema species in Hungary, a Hungarian-Italian cooperation has been started. This work presents a detailed analysis of the distribution and morphometrical data and associated host plants of the Xiphinema americanum-group species occurring in Hungary.

\section{Material and methods}

During a survey started in August 1998 and continued in 2004 (from June until November) and 2005 (from April until October), a total of 272 samples were taken from the vicinity of 53 localities, from the rhizosphere of 70 plant species. Soil samples were taken from the top 20-40 cm of soil using a spade of $25 \mathrm{~cm}$ in width. One bulk sample comprised of soil taken from around at least 2 plants. Nematodes were extracted from subsamples of 200 to approx. $500 \mathrm{~g}$ using decanting and sieving technique with 1010 and $100 \mu \mathrm{m}$ sieves (Flegg, 1967). Longidorid nematodes were picked out from the suspensions, fixed in hot formalin or FP 4:1, processed and mounted in anhydrous glycerine. Measurements were made using a light microscope with a camera lucida. 
Mean and range of the measured values were recorded for several morphometrical characters relevant for determination of the encountered species (e.g. Barsi, 1994; Kumari, 2006; Lamberti et al., 2000, 2004; Luc et al., 1998).

The Hungarian specimens were compared with measurements of the topotypes of X. brevicollum (Luc et al., 1998) and original type material of $X$. pachtaicum and $X$. simile. UTM maps were prepared with ArcView GIS Version 3.1 and scatterplot graphs created with Harvard Graphics 98 software.

\section{Results}

Species belonging to the $X$. americanum-group were present in the samples with the following proportions: $X$. brevicollum $(4.41 \%)$, X. pachtaicum $(3.67 \%)$ and $X$. simile $(4.77 \%)$. The distribution of these species is rather scattered throughout the country (Fig. 1)

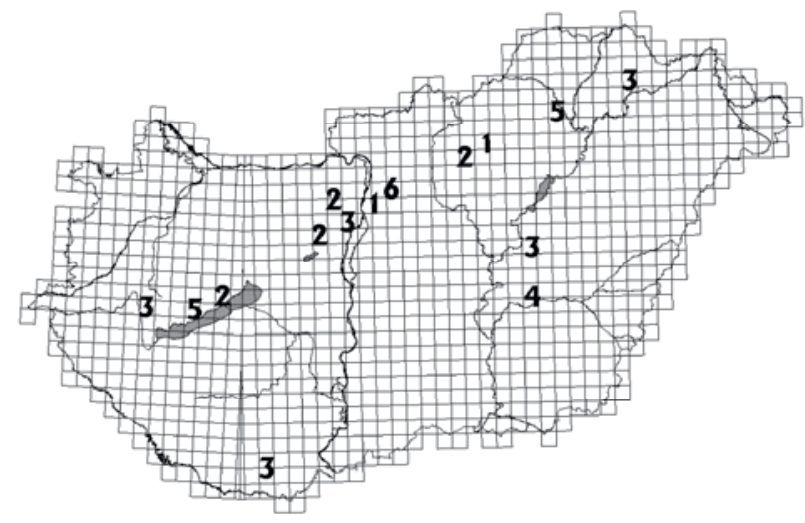

Fig. 1. UTM map to display occurence data for species belonging to the Xiphinema americanum group (Xiphinema brevicollum, Xiphinema pachtaicum and Xiphinema simile) in Hungary. 1- site infested with Xiphinema brevicollum; 2 - site infested with Xiphinema simile; 3 - site infested with Xiphinema pachtaicum;

4 - site infested with Xiphinema brevicollum and Xiphinema simile; 5 - site infested with Xiphinema brevicollum and Xiphinema pachtaicum; 6 - site infested with all the three species

\section{Xiphinema brevicollum (Table 2)}

Female: Habitus coiled in a closed $\mathrm{C}$ when killed; body robust, cylindrical, tapering gradually towards the extremities; cuticle very finely transversely striated. Labial region hemi-elliptical $5-5.5 \mu \mathrm{m}$ high, separated from the rest of the body by a depression. Amphid stirrup-shaped; odontostyle robust $1.5-1.7 \mu \mathrm{m}$ in diameter at the tip and $2.5-2.7 \mu \mathrm{m}$ just above the forked junction with the well flanged odontophore; guiding ring typical of the genus; oesophagus dorylaimoid with the anterior part tubular, bearing refringent mucro at $52-55 \mu \mathrm{m}$ from base of the odontophore; basal bulb approximately $1 / 3$ of the total oesophagus length, containing three glandular nuclei. Female reproductive system amphidelphic with equally developed opposed branches; vulva slit-like; vagina occupying $1 / 3$ of the corresponding body diameter; no spermatheca, ovaries reflexed. Tail short, conoid with rounded termi- nus and two pairs of caudal pores.

Male: one male was found in the Kisnána isolate. Male similar to females, but body more curved posteriorly. One adanal pair and 7 ventromedian precloacal supplements present. Spicules slightly curved, measuring $53.4 \mu \mathrm{m}$ along the arch, lateral guiding pieces $14.5 \mu \mathrm{m}$ long.

Juveniles: clearly separated into four stages based on the stylet measurements (Fig. 2) and morphologically similar to females but tails are longer and more tapering (Table 2).

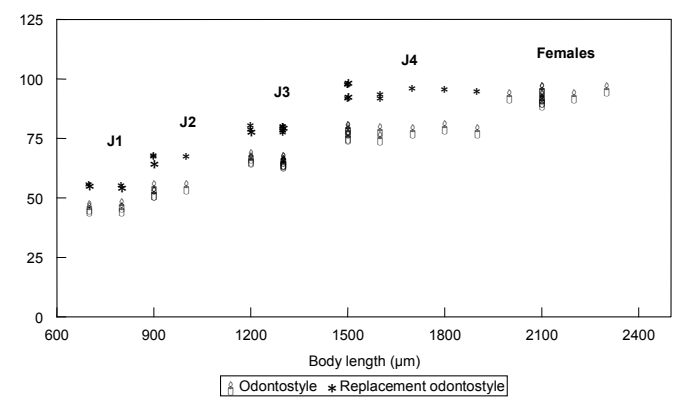

Fig. 2. Scatter diagram separating juveniles and females of Xiphinema brevicollum, in the function of the stylet measurements $(\mu \mathrm{m})$

Xiphinema brevicollum has been recovered from 12 samples in six localities (Fig. 1) Localities and plants associated are given in Table 1.

\section{Xiphinema pachtaicum (Table 3)}

Female: Habitus a single spiral or closed $\mathrm{C}$ when relaxed. Body gradually tapering towards the extremities; cuticle finely striated transversely. Labial region $3.5-4.0 \mu \mathrm{m}$ high, expanded, frontally flattened, laterally rounded, separated from the rest of the body by a deep constriction. Amphid stirrup-shaped. Odontostyle, odontophore and guiding ring sheath typical of the genus. Oesophagus dorylaimoid. Oesophageal bulb $1 / 3-1 / 4$ of total length. Vulva posterior to median position, between $52.5-58.5 \%$, vagina extending to $1 / 2$ of corresponding body diameter. Gonads paired, opposed and reflexed; spermatheca and " $Z$ " organ absent. Tail 28.7 - $33.3 \mu \mathrm{m}$ long, conical, dorsally convex gradually tapering towards the extremity with pointed end. Two pairs of caudal pores.

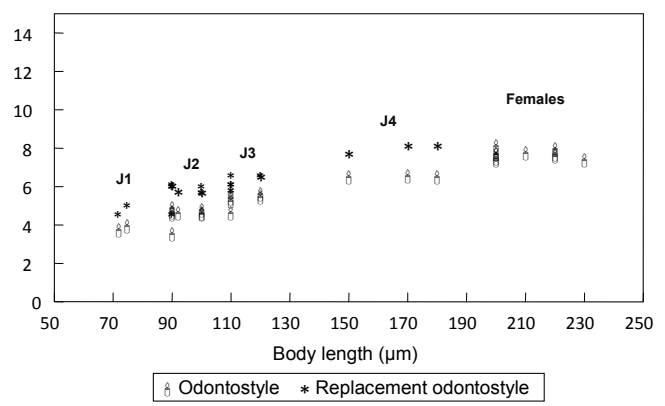

Fig. 3. Scatter diagram separating juveniles and females of Xiphinema pachtaicum, in the function of the stylet measurements $(\mu \mathrm{m})$ 
Table 1. The distribution (with UTM-codes in brackets) and plant associations of the $X$. americanum group species in Hungary

\begin{tabular}{|c|c|c|c|}
\hline & X. brevicollum & X. pachtaicum & $X$. simile \\
\hline Vitis vinifera $L$. & $\begin{array}{c}\text { Badacsony (XM98), Kisnána } \\
\text { (DU30) }\end{array}$ & $\begin{array}{c}\text { Badacsony (XM98), Olaszliszka (EU } \\
\text { 34), Villány (BR 98) }\end{array}$ & $\begin{array}{l}\text { Telki (CT 36), Tordas } \\
\text { (CT 24) }\end{array}$ \\
\hline Prunus armeniaca L. & $\begin{array}{c}\text { Gödöllö (CT 77), Kisnána (DU } \\
\text { 30) }\end{array}$ & & Tordas (CT 24) \\
\hline Prunus avium $L$. & Gödöllő (CT77) & $\begin{array}{c}\text { Felsőzsolca (DU 82), Nagykörü (DT } \\
63 \text { ) }\end{array}$ & Csopak (YN 10) \\
\hline Prunus cerasus L. & & Érd-Elviramajor (CT 35) & Tordas (CT 24) \\
\hline Prunus domestica L. & & & Tordas (CT 24) \\
\hline Pyrus communis L. & Gödöllő (CT 77) & & \\
\hline $\begin{array}{l}\text { Malus domesticus L. \& Rubus } \\
\text { caesius L. }\end{array}$ & Felsőzsolca (DU 82) & & \\
\hline Juglans regia L. & Kisnána (DU 30) & & \\
\hline Amygdalus communis L. & & & Tordas (CT 24) \\
\hline Cydonia oblonga Miller & Gödöllő (CT 77) & & Gödöllő (CT 77) \\
\hline Rosa indica Lindl & Budapest (CT 56) & & \\
\hline Rosa canina L. & & & Abasár (DT 29) \\
\hline Platanus sp. & $\begin{array}{c}\text { Gödöllő (CT 77), Szarvas (DS } \\
69) \\
\end{array}$ & & \\
\hline Acer sp. & & Gödöllő (CT 77) & Gödöllő (CT 77) \\
\hline Betula pendula Roth & & & Gödöllő (CT 77) \\
\hline Pinus nigra Arnold & & Gödöllő (CT 77) & \\
\hline Robinia pseudo-acacia L. & & $\begin{array}{c}\text { Érd-Elviramajor (CT 35), Zalaszántó } \\
\text { (XM 69) }\end{array}$ & \\
\hline Sorbus torminalis L. (Cr.) & & & Szarvas (DS 69) \\
\hline
\end{tabular}

Table 2. Morphometric data of a Xiphinema brevicollum isolate from Hungary. All measurements are in $\mu \mathrm{m}$ except for body length (L, in mm) and in the form: mean and range of the measured values.

\begin{tabular}{|c|c|c|c|c|c|c|}
\hline \multirow{2}{*}{$\begin{array}{l}\text { Locality } \\
\text { Host } \\
\mathrm{n}\end{array}$} & \multicolumn{6}{|c|}{ Kisnána } \\
\hline & 10 운 & 10 & $4 \mathrm{~J} 1$ & $4 \mathrm{~J} 2$ & $10 \mathrm{~J} 3$ & $10 \mathrm{~J} 4$ \\
\hline \multirow[t]{2}{*}{$\mathrm{L}$} & $2.1 \pm 0.07$ & 2.1 & $0.77 \pm 0.05$ & $0.95 \pm 0.04$ & $1.3 \pm 0.05$ & $1.6 \pm 0.16$ \\
\hline & $2.0-2.3$ & & $0.72-0.83$ & $0.92-1.00$ & $1.2-1.3$ & $1.5-1.9$ \\
\hline \multirow[t]{2}{*}{ A } & $47.7 \pm 1.24$ & 45.6 & $38.7 \pm 1.06$ & $38.7 \pm 2.63$ & $41.0 \pm 1.50$ & $44.4 \pm 2.58$ \\
\hline & $45-49.4$ & & $37.9-40.3$ & $36-41.7$ & $37.7-42.6$ & $40-49.3$ \\
\hline \multirow[t]{2}{*}{$\mathrm{B}$} & $6.2 \pm 0.34$ & 6.6 & $4 \pm 0.19$ & $4.1 \pm 0.33$ & $5.2 \pm 0.43$ & $5.2 \pm 0.34$ \\
\hline & $5.7-6.9$ & & $3.8-4$ & $3.8-4.5$ & $4.5-5.7$ & $4.8-5.8$ \\
\hline \multirow[t]{2}{*}{$\mathrm{C}$} & $82.2 \pm 5.62$ & 76 & $20.7 \pm 1.38$ & $27.8 \pm 1.58$ & $37 \pm 3.41$ & $51.7 \pm 4.99$ \\
\hline & $71.8-90.5$ & & $19.5-22.7$ & $25.8-29.7$ & $30.2-41.8$ & $45.6-59.8$ \\
\hline \multirow[t]{2}{*}{$c^{\prime}$} & $0.9 \pm 0.03$ & 0.9 & $2.8 \pm 0.08$ & $2.1 \pm 0.14$ & $1.6 \pm 0.11$ & $1.2 \pm 0.10$ \\
\hline & $0.9-1.0$ & & $2.7-2.9$ & $2-2.3$ & $1.5-1.8$ & $1.1-1.4$ \\
\hline \multirow[t]{2}{*}{$\mathrm{V}$} & $49.5 \pm 1.09$ & --- & --- & --- & --- & --- \\
\hline & $47.4-50.7$ & & & & & \\
\hline \multirow[t]{2}{*}{ Odontostyle $\mu \mathrm{m}$} & $93.9 \pm 2.08$ & 96.6 & $46.6 \pm 0.66$ & $54 \pm 1.33$ & $66.4 \pm 1.41$ & $78.5 \pm 1.56$ \\
\hline & $90.8-96.6$ & & $46-47$ & $53-55$ & $63.2-68.4$ & $76-80.5$ \\
\hline \multirow[t]{2}{*}{ Odontophore $\mu \mathrm{m}$} & $58.2 \pm 1.79$ & 58 & $32.6 \pm 1.36$ & $38.8 \pm 2.12$ & $45 \pm 1.39$ & $51.5 \pm 0.48$ \\
\hline & $56.3-60.9$ & & $31.6-34.5$ & $37.4-42$ & $43-47$ & $50.6-51.7$ \\
\hline \multirow[t]{2}{*}{ Replacement odontostyle } & --- & --- & $55.3 \pm 0.55$ & $66.8 \pm 1.27$ & $79 \pm 0.99$ & $95 \pm 2.46$ \\
\hline & & & $54.6-55.7$ & $65-67.8$ & $77.6-80.5$ & $92-98.9$ \\
\hline \multirow[t]{2}{*}{ Oral aperture to basal guide ring } & $79 \pm 1.12$ & 77 & $37.2 \pm 0.98$ & $44.4 \pm 1.09$ & $54.5 \pm 1.23$ & $64 \pm 2.41$ \\
\hline & $77-81$ & & $36.2-38.5$ & $43.7-46$ & $51.7-55.7$ & $61-67.8$ \\
\hline \multirow[t]{2}{*}{ Tail } & $25.8 \pm 2.32$ & 27.6 & $37 \pm 0.33$ & $34.2 \pm 1.33$ & $33.4 \pm 1.42$ & $30.9 \pm 1.18$ \\
\hline & $23.6-31.6$ & & $36.8-37$ & $33.3-35.6$ & $31.6-35.6$ & $28.7-31.6$ \\
\hline \multirow[t]{2}{*}{ Hyaline portion of tail $(\mathrm{J})$} & $9.1 \pm 0.89$ & 7 & $6.8 \pm 0.55$ & $7.2 \pm 0.33$ & $8.8 \pm 0.47$ & $9.1 \pm 0.50$ \\
\hline & $7.5-10$ & & $6.3-7.5$ & $6.9-7.5$ & $8.0-9.8$ & $8.6-9.8$ \\
\hline \multirow[t]{2}{*}{ Body diam. at lip region } & $13.3 \pm 0.42$ & 13.8 & $7.8 \pm 0.33$ & $8.6 \pm 0.00$ & $10.0 \pm 0.40$ & $11.4 \pm 0.61$ \\
\hline & $12.6-13.8$ & & $7.5-8$ & $8.6-8.6$ & $9.2-10$ & $10.9-12.6$ \\
\hline \multirow[t]{2}{*}{ Body diam. at basal guide ring } & $31.2 \pm 0.77$ & 32.8 & $16.2 \pm 0.72$ & $19 \pm 0.55$ & $22.6 \pm 0.55$ & $27 \pm 1.67$ \\
\hline & $30.5-32.8$ & & $15.5-17$ & $18.4-19.5$ & $21.8-23.6$ & $24.7-30$ \\
\hline \multirow[t]{2}{*}{ Body diam. at base of oesophagus } & $39.5 \pm 1.32$ & 40 & $18.5 \pm 0.72$ & $23 \pm 1.41$ & $28.5 \pm 1.12$ & $34.2 \pm 2.20$ \\
\hline & $37.4-42$ & & $17.8-19.5$ & $21.3-24.7$ & $27-30.5$ & $31.6-37.4$ \\
\hline \multirow[t]{2}{*}{ Body diam. at mid-body or vulva } & $44.3 \pm 1.35$ & 46 & $19.8 \pm 0.74$ & $24.6 \pm 1.44$ & $30.5 \pm 1.34$ & $36.4 \pm 2.25$ \\
\hline & $42-46.6$ & & $19.0-20.7$ & $23-26.4$ & $28.7-32.8$ & $34.5-40$ \\
\hline \multirow[t]{2}{*}{ Body diam. at anus } & $27.8 \pm 2.12$ & 29.3 & $13.2 \pm 0.47$ & $16.2 \pm 0.72$ & $20.5 \pm 0.82$ & $25.3 \pm 1.79$ \\
\hline & $26-32$ & & $12.6-13.8$ & $15.5-17$ & $19-21.8$ & $23.6-28.7$ \\
\hline \multirow[t]{2}{*}{ Body diam. at beginning of $\mathrm{J}$} & $15.5 \pm 0.98$ & 14.5 & $5.3 \pm 0.29$ & $6.3 \pm 0.47$ & $8.5 \pm 0.36$ & $12.8 \pm 0.74$ \\
\hline & $13.8-16.7$ & & $5.2-5.7$ & $5.7-6.9$ & $8-9$ & $11.5-13.8$ \\
\hline Spicules & & 53.5 & & & & \\
\hline
\end{tabular}

$\mathrm{n}=$ number of specimens; $\mathrm{A}=$ body length / greatest body diameter; $\mathrm{B}=$ body length / distance from anterior to esophago-intestinal valve;

$\mathrm{C}=$ body length / tail length; $\mathrm{c}^{\prime}=$ tail length/anal body diameter; $\mathrm{V}=\%$ distance of vulva from anterior 
Table 3. Morphometrics of three isolates of Xiphinema pachtaicum from Hungary. All measurements are in $\mu \mathrm{m}$ except for body length (L, in mm) and in the form: mean and range of the measured values.

\begin{tabular}{|c|c|c|c|c|c|c|c|}
\hline $\begin{array}{l}\text { Locality } \\
\text { Host }\end{array}$ & & & $\begin{array}{l}\text { Gödöllö } \\
\text { Maple } \\
\text { (Acer sp.) }\end{array}$ & & & $\begin{array}{c}\text { Érd } \\
\text { Sour cherry } \\
\text { (Prunus cerasus) }\end{array}$ & $\begin{array}{c}- \\
\text { Grapevine } \\
\text { (Vitis vinifera) }\end{array}$ \\
\hline $\mathrm{n}$ & 10 우 & $3 \mathrm{~J} 1$ & $8 \mathrm{~J} 2$ & $5 \mathrm{~J} 3$ & $3 \mathrm{~J} 4$ & 10 우 & 5 우 \\
\hline $\mathrm{L}$ & $\begin{array}{c}2.1 \pm 0.13 \\
2.0-2.3\end{array}$ & $\begin{array}{l}0.79 \pm 0.11 \\
0.72-0.92\end{array}$ & $\begin{array}{c}0.98 \pm 0.06 \\
0.92-1.1\end{array}$ & $\begin{array}{l}1.2 \pm 0.05 \\
1.1-1.2\end{array}$ & $\begin{array}{l}1.6 \pm 0.18 \\
1.4-1.8\end{array}$ & $\begin{array}{l}1.8 \pm 0.10 \\
1.6-1.9\end{array}$ & $\begin{array}{l}1.9 \pm 0.11 \\
1.7-2.0\end{array}$ \\
\hline A & $\begin{array}{l}65.7 \pm 3.04 \\
62.5-70.9\end{array}$ & $\begin{array}{l}45.0 \pm 3.41 \\
41.7-48.5\end{array}$ & $\begin{array}{l}47.6 \pm 2.63 \\
43.2-51.4\end{array}$ & $\begin{array}{l}53.6 \pm 2.98 \\
48.8-55.7\end{array}$ & $\begin{array}{l}62.7 \pm 3.63 \\
59.5-66.7\end{array}$ & $\begin{array}{c}69.5 \pm 3.24 \\
64-74\end{array}$ & $\begin{array}{c}66 \pm 3.29 \\
62.7-69.8\end{array}$ \\
\hline B & $\begin{array}{c}7.5 \pm 0.95 \\
6.2-8.9\end{array}$ & $\begin{array}{c}4.8 \pm 0.58 \\
4.2-5.3\end{array}$ & $\begin{array}{c}4.6 \pm 0.55 \\
4.0-5.5\end{array}$ & $\begin{array}{c}5.2 \pm 0.17 \\
5.0-5.4\end{array}$ & $\begin{array}{c}6.2 \pm 0.23 \\
6.0-6.5\end{array}$ & $\begin{array}{c}6.4 \pm 0.40 \\
6-7\end{array}$ & $\begin{array}{c}6.4 \pm 0.62 \\
5.5-7\end{array}$ \\
\hline $\mathrm{C}$ & $\begin{array}{c}68.5 \pm 4.81 \\
61.8-76\end{array}$ & $\begin{array}{c}26.6 \pm 4.74 \\
23.2-32\end{array}$ & $\begin{array}{c}28.8 \pm 1.72 \\
26.2-31\end{array}$ & $\begin{array}{l}34.1 \pm 1.49 \\
32.3-36.4\end{array}$ & $\begin{array}{l}47.4 \pm 3.08 \\
43.9-49.2\end{array}$ & $\begin{array}{c}63.2 \pm 4.15 \\
55-69.8\end{array}$ & $\begin{array}{l}59.4 \pm 5.02 \\
53.4-64.2\end{array}$ \\
\hline$C^{\prime}$ & $\begin{array}{c}1.7 \pm 0.07 \\
1.5-1.8\end{array}$ & $\begin{array}{c}2.8 \pm 0.03 \\
2.8-2.8\end{array}$ & $\begin{array}{l}2.5 \pm 0.15 \\
2.3-2.8\end{array}$ & $\begin{array}{l}2.5 \pm 0.13 \\
2.3-2.6\end{array}$ & $\begin{array}{c}2.0 \pm 0.06 \\
1.9-2.0\end{array}$ & $\begin{array}{l}1.9 \pm 0.10 \\
1.7-2\end{array}$ & $\begin{array}{l}1.8 \pm 0.08 \\
1.8-1.9\end{array}$ \\
\hline V & $\begin{array}{l}53 \pm 0.81 \\
52.5-55\end{array}$ & --- & --- & --- & --- & $\begin{array}{c}55.6 \pm 1.40 \\
54-58.5\end{array}$ & $\begin{array}{c}56.8 \pm 0.92 \\
56-58\end{array}$ \\
\hline Odontostyle & $\begin{array}{c}77.8 \pm 2.38 \\
74.7-82\end{array}$ & $\begin{array}{c}38.3 \pm 2.02 \\
36.2-40\end{array}$ & $\begin{array}{l}47.6 \pm 1.21 \\
46.6-50.0\end{array}$ & $\begin{array}{l}55.9 \pm 1.74 \\
53.4-58.0\end{array}$ & $\begin{array}{l}66.3 \pm 0.33 \\
66.1-66.7\end{array}$ & $\begin{array}{l}79.2 \pm 1.46 \\
77.6-81.6\end{array}$ & $\begin{array}{c}86.7 \pm 1.49 \\
85-89\end{array}$ \\
\hline Odontophore & $\begin{array}{c}51.6 \pm 1.58 \\
49.4-53\end{array}$ & $\begin{array}{l}30.7 \pm 2.02 \\
28.7-32.8\end{array}$ & $\begin{array}{l}36.9 \pm 1.56 \\
35.1-39.7\end{array}$ & $\begin{array}{l}38.7 \pm 1.44 \\
37.4-40.2\end{array}$ & $\begin{array}{l}48.1 \pm 2.02 \\
46.0-50.0\end{array}$ & $\begin{array}{c}43 \pm 0.0 .90 \\
42-44.8\end{array}$ & $\begin{array}{c}48.3 \pm 2.11 \\
44.8-50\end{array}$ \\
\hline $\begin{array}{l}\text { Replacement } \\
\text { odontostyle }\end{array}$ & --- & $\begin{array}{c}46.9 \pm 2.65 \\
45.4-50\end{array}$ & $\begin{array}{c}59.1 \pm 1.68 \\
57-61.5\end{array}$ & $\begin{array}{c}64.3 \pm 2.52 \\
61.5-66\end{array}$ & $\begin{array}{c}80.7 \pm 2.65 \\
77.6-82\end{array}$ & --- & --- \\
\hline $\begin{array}{l}\text { Oral aperture to basal } \\
\text { guide ring }\end{array}$ & $\begin{array}{l}66.6 \pm 0.96 \\
64.9-67.8\end{array}$ & $\begin{array}{l}32.6 \pm 0.88 \\
31.6-33.3\end{array}$ & $\begin{array}{l}40.4 \pm 1.01 \\
38.5-42.0\end{array}$ & $\begin{array}{l}49.4 \pm 0.81 \\
48.9-50.6\end{array}$ & $\begin{array}{l}57.1 \pm 0.88 \\
56.3-58.0\end{array}$ & $\begin{array}{c}68.8 \pm 2.26 \\
66-71.8\end{array}$ & $\begin{array}{l}76 \pm 2.01 \\
74-79.3\end{array}$ \\
\hline Tail & $\begin{array}{l}30.8 \pm 1.49 \\
28.7-32.8\end{array}$ & $\begin{array}{l}30 \pm 1.85 \\
28.7-32\end{array}$ & $\begin{array}{l}34.0 \pm 1.83 \\
31.6-36.2\end{array}$ & $\begin{array}{l}34.5 \pm 2.19 \\
31.6-36.8\end{array}$ & $\begin{array}{l}34.7 \pm 1.76 \\
32.8-36.2\end{array}$ & $\begin{array}{c}29 \pm 0.82 \\
27.6-30.5\end{array}$ & $\begin{array}{l}31.6 \pm 1.22 \\
30.5-33.3\end{array}$ \\
\hline $\begin{array}{l}\text { Hyaline portion } \\
\text { of tail }(\mathrm{J})\end{array}$ & $\begin{array}{c}8.4 \pm 0.50 \\
7.5-9.2\end{array}$ & $\begin{array}{c}5.0 \pm 0.66 \\
4.6-5.7\end{array}$ & $\begin{array}{c}5.8 \pm 0.20 \\
5.7-6.3\end{array}$ & $\begin{array}{c}5.2 \pm 0.00 \\
5.2-5.2\end{array}$ & $\begin{array}{c}6.7 \pm 0.33 \\
6.3-6.9\end{array}$ & $\begin{array}{c}8 \pm 0.61 \\
7-8.6\end{array}$ & $\begin{array}{c}9.2 \pm 0.41 \\
8.6-9.8\end{array}$ \\
\hline $\begin{array}{l}\text { Body diam. at lip } \\
\text { region }\end{array}$ & $\begin{array}{l}9.5 \pm 0.28 \\
9.2-9.8\end{array}$ & $\begin{array}{c}6.9 \pm 0.00 \\
6.9-6.9\end{array}$ & $\begin{array}{l}7.5 \pm 0.00 \\
7.5-7.5\end{array}$ & $\begin{array}{l}7.9 \pm 0.48 \\
7.5-8.6\end{array}$ & $\begin{array}{l}8.6 \pm 0.00 \\
8.6-8.6\end{array}$ & $\begin{array}{l}8.8 \pm 0.29 \\
8.6-9\end{array}$ & $\begin{array}{l}8.5 \pm 0.26 \\
8-8.6\end{array}$ \\
\hline $\begin{array}{l}\text { Body diam. at basal } \\
\text { guide ring }\end{array}$ & $\begin{array}{c}21.6 \pm 1.02 \\
20-23.6\end{array}$ & $\begin{array}{c}14.0 \pm 0.33 \\
13.8-14\end{array}$ & $\begin{array}{l}15.4 \pm 0.59 \\
14.5-16.1\end{array}$ & $\begin{array}{l}17.0 \pm 0.51 \\
16.7-17.8\end{array}$ & $\begin{array}{l}18.8 \pm 0.88 \\
17.8-19.5\end{array}$ & $\begin{array}{c}18.8 \pm 0.75 \\
17.8-20\end{array}$ & $\begin{array}{c}21.3 \pm 0.81 \\
20-22.5\end{array}$ \\
\hline $\begin{array}{l}\text { Body diam. at base } \\
\text { of oesophagus }\end{array}$ & $\begin{array}{l}27.6 \pm 1.03 \\
25.9-28.7\end{array}$ & $\begin{array}{c}16.3 \pm 0.88 \\
15.5-17\end{array}$ & $\begin{array}{l}18.9 \pm 0.65 \\
17.8-19.5\end{array}$ & $\begin{array}{l}20.2 \pm 1.59 \\
18.4-21.8\end{array}$ & $\begin{array}{l}24.3 \pm 2.02 \\
22.5-26.4\end{array}$ & $\begin{array}{c}22.5 \pm 1.05 \\
20.7-24\end{array}$ & $\begin{array}{c}24.5 \pm 1.61 \\
23-26.5\end{array}$ \\
\hline $\begin{array}{l}\text { Body diam. at mid-body } \\
\text { or vulva }\end{array}$ & $\begin{array}{l}32.1 \pm 1.14 \\
31.0-34.5\end{array}$ & $\begin{array}{c}17.6 \pm 1.20 \\
16.7-19\end{array}$ & $\begin{array}{c}20.5 \pm 0.67 \\
19.5-21\end{array}$ & $\begin{array}{l}22.0 \pm 1.59 \\
20.1-23.6\end{array}$ & $\begin{array}{c}26.2 \pm 2.32 \\
24-28.7\end{array}$ & $\begin{array}{l}26.4 \pm 1.25 \\
24.7-28.2\end{array}$ & $\begin{array}{c}28.5 \pm 2.65 \\
26-31.6\end{array}$ \\
\hline Body diam. at anus & $\begin{array}{l}18.4 \pm 0.69 \\
17.8-19.5\end{array}$ & $\begin{array}{c}10.7 \pm 0.66 \\
10-11.5\end{array}$ & $\begin{array}{l}13.6 \pm 0.68 \\
12.6-14.5\end{array}$ & $\begin{array}{l}13.7 \pm 0.63 \\
12.6-14.5\end{array}$ & $\begin{array}{c}17.6 \pm 1.45 \\
16-19\end{array}$ & $\begin{array}{c}15.7 \pm 0.81 \\
15-17\end{array}$ & $\begin{array}{l}17 \pm 0.26 \\
16.7-17\end{array}$ \\
\hline $\begin{array}{l}\text { Body diam. } \\
\text { at beginning of } \mathrm{J}\end{array}$ & $\begin{array}{c}8.2 \pm 0.53 \\
7.5-9\end{array}$ & $\begin{array}{c}4.6 \pm 0.57 \\
4-5\end{array}$ & $\begin{array}{c}5.0 \pm 0.30 \\
4.6-5.2\end{array}$ & $\begin{array}{c}5.7 \pm 0.00 \\
5.7-5.7\end{array}$ & $\begin{array}{c}7.1 \pm 0.33 \\
6.9-7.5\end{array}$ & $\begin{array}{l}7 \pm 0.35 \\
6.5-7.5\end{array}$ & $\begin{array}{c}7.6 \pm 0.48 \\
7-8\end{array}$ \\
\hline
\end{tabular}

$\mathrm{n}=$ number of specimens; $\mathrm{a}=$ body length / greatest body diameter; $\mathrm{b}=$ body length / distance from anterior to esophago-intestinal valve; $\mathrm{c}=$ body length / tail length; $\mathrm{c}^{\prime}=$ tail length/anal body diameter; $\mathrm{V}=\%$ distance of vulva from anterior

Male not found.

Juveniles resemble females except for body posture i. e. less ventrally curved than adults. Four juvenile stages were distinguished (Tab. 3, Fig. 3).

The three isolates of $X$. pachtaicum measured in details (Tab. 3) are basically similar for most morphological and morphometric characters. However, the Gödöllő isolate compared to the other two has longer body $(2.1 \mathrm{~mm} \mathrm{vs} 1.8$ $\mathrm{mm})$, shorter odontostyle $(77.8 \mu \mathrm{m}$ vs $79.2-86.7 \mu \mathrm{m})$, anterior $\mathrm{V}(\mathrm{V}=53$ vs $56-57 \%)$, more anterior position of guiding ring $(66.6 \mu \mathrm{m} v s 68.8 \mu \mathrm{m})$.

Xiphinema pachtaicum has been found in 10 samples from 8 known localities (Fig. 1) and one unknown locality.

\section{Xiphinema simile (Table 4)}

Female: Habitus a single spiral or closed $\mathrm{C}$ when killed; body tapering gradually towards the extremities; cuticle very finely striated transversely, $1-1.65 \mu \mathrm{m}$ thick along the body. Labial region expanded, 3.5 to 4.6 high, separated from the rest of the body by a deep constriction. Amphideal pouches stirrup-shaped. Odontostyle, odontophore and guiding sheath typical of the genus. Oesophagus dorylaimoid. Oesophagus basal bulb 72-86 $\mu \mathrm{m}$ long and $13-15 \mu \mathrm{m}$ wide. Vulva shortly posterior to mid-body, 53 - $56 \%$; vagina about $1 / 2$ to $1 / 3$ of the corresponding body diameter. Gonads amphidelphic, reflexed with no particular characters in their components. Prerectum not visible; rectum $16-25 \mu \mathrm{m}$ long i. e. similar to anal body diameter $(17.2-20.0 \mu \mathrm{m})$. Tail conoid with subacute terminus. Two pairs of caudal pores.

Male not found.

Juveniles were not selected for measurements.

Most of the morphometric characters of $X$. simile (Table 4) found at Gödöllö in the rhizosphere of maple (Acer sp.) and in a grapevine sample from an unknown locality are biometrically identical.

Xiphinema simile has been recovered from 13 samples in six known localities (Fig. 1) and one unknown locality. 
Table 4. Morphometrics of two isolates of Xiphinema simile from Hungary. All measurements are in $\mu \mathrm{m}$ except for body length $(\mathrm{L}$, in $\mathrm{mm}$ ) and in the form: mean and range of the measured values.

\begin{tabular}{|c|c|c|}
\hline Locality & Gödöllő & - \\
\hline Host & $\begin{array}{c}\text { Maple (Acer } \\
\text { sp.) }\end{array}$ & Grapevine (Vitis vinifera) \\
\hline $\mathrm{n}$ & 10 우 & 10 웅 \\
\hline \multirow[t]{2}{*}{$\mathrm{L}$} & $2.3 \pm 0.13$ & $2.3 \pm 0.09$ \\
\hline & $2.1-2.5$ & $2.1-2.4$ \\
\hline \multirow[t]{2}{*}{ A } & $78.8 \pm 3.19$ & $75.7 \pm 3.15$ \\
\hline & $74-81$ & $71.3-79.8$ \\
\hline \multirow[t]{2}{*}{ B } & $8.4 \pm 1.08$ & $7.8 \pm 0.28$ \\
\hline & $7.4-9.6$ & $7.4-8.3$ \\
\hline \multirow[t]{2}{*}{$\mathrm{C}$} & $77.3 \pm 2.30$ & $72.6 \pm 4.39$ \\
\hline & $74-80$ & $67-79.8$ \\
\hline \multirow[t]{2}{*}{$c^{\prime}$} & $1.6 \pm 0.09$ & $1.7 \pm 0.09$ \\
\hline & $1.5-1.8$ & $1.6-1.8$ \\
\hline \multirow[t]{2}{*}{$\mathrm{V}$} & $55 \pm 0.75$ & $55 \pm 0.87$ \\
\hline & $54-56$ & $53-56$ \\
\hline \multirow[t]{2}{*}{ Odontostyle } & $67.5 \pm 1.93$ & $66.9 \pm 1.39$ \\
\hline & $66.1-70$ & $64.4-68.4$ \\
\hline \multirow[t]{2}{*}{ Odontophore } & $44.1 \pm 1.11$ & $42 \pm 1.88$ \\
\hline & $43.1-45.9$ & $38.5-44$ \\
\hline Oral aperture & $60.2 \pm 1.25$ & $58 \pm 2.19$ \\
\hline to guide ring & $58.6-62$ & $54.6-61$ \\
\hline \multirow[t]{2}{*}{ Tail } & $29.6 \pm 1.55$ & $31 \pm 0.99$ \\
\hline & $27.6-31.6$ & $29.3-32$ \\
\hline Hyaline portion & $6.2 \pm 0.48$ & $6 \pm 0.67$ \\
\hline of tail $(\mathrm{J})$ & $5.7-6.9$ & $5.2-6.9$ \\
\hline Body diam. & $9.4 \pm 0.51$ & $9 \pm 0.40$ \\
\hline at lip region & $9.2-10$ & $8.6-9.8$ \\
\hline Body diam. & $19.4 \pm 0.75$ & $19.4 \pm 0.67$ \\
\hline at guide ring & $18.4-20.1$ & $18.4-20.5$ \\
\hline Body diam. & $24.7 \pm 0.70$ & $25.4 \pm 1.71$ \\
\hline at base & $23.6-25.3$ & $23-28.7$ \\
\hline \multicolumn{3}{|l|}{ of oesophagus } \\
\hline Body diam. & $29.1 \pm 1.12$ & $29.8 \pm 1.14$ \\
\hline at vulva & $27.6-30.5$ & $28.2-31.6$ \\
\hline Body diam. & $18.2 \pm 0.87$ & $17.8 \pm 0.96$ \\
\hline at anus & $17.2-19.5$ & $17.2-20$ \\
\hline Body diam. & $7.8 \pm 0.31$ & $7.3 \pm 0.47$ \\
\hline at beginning of $\mathrm{J}$ & $7.5-8$ & $7-8$ \\
\hline
\end{tabular}

$\mathrm{n}=$ number of specimens; $\mathrm{a}=$ body length / greatest body diameter; $\mathrm{b}=$ body length / distance from anterior to esophago-intestinal valve; $\mathrm{c}=$ body length / tail length; c'= tail length/anal body diameter; $\mathrm{V}=\%$ distance of vulva from anterior

\section{Discussion}

\section{Xiphinema brevicollum}

Xiphinema brevicollum was first described as $X$. brevicolle Lordello \& Da Costa, 1961 and later synonymized with $X$. taylori Lamberti, Ciancio, Agostinelli \& Coiro, 1991 by Luc et al. (1998) that have modified the specific epithete in X. brevicollum.

The morphometrics of the Hungarian isolate are in agreement with the original description of the species, but when compared with the topotypes (Luc et al., 1998), this Hungarian isolate has a longer body (2.1 vs $1.9 \mathrm{~mm})$; and higher 'c' values (82 vs 77), shorter odontostyle (94 vs. $101 \mu \mathrm{m}$ ), a more anterior position of guiding ring (79 vs $86 \mu \mathrm{m})$; and vulva $(\mathrm{V}=49.5$ vs $53 \%)$. To resolve this contradiction, molecular methods (Lazarova et al., 2006; Oliveira et al., 2005) will have to be involved in future studies.

In agreement with Jenser (1985), we found X. brevicollum on sour cherry. Xiphinema brevicollum was found associated with cherry, apricot, apple, quince, rose, sycamore and pear, representing new plant association records for Hungary.

\section{Xiphinema pachtaicum}

The three isolates of $X$. pachtaicum found in the rhizosphere of maple at Gödöllö, in the rhizosphere of sour cherry at Érd-Elviramajor and with grapevine from an unknown locality revealed some differences in the morphometric characters when compared with former descriptions. The Gödöllö isolate has a longer body (2.1 vs 1.8 and $1.9 \mathrm{~mm}$ resp.), shorter odontostyle, (77.8 vs 79.2 and $86.7 \mu \mathrm{m})$, more anterior vulva $(\mathrm{V}=53$ vs 55.6 and $56.87 \%)$ and guiding ring (66.6 vs 68.8 and $76.7 \mu \mathrm{m}$ distance from anterior extremity). $X$. pachtaicum was thoroughly described and illustrated by Lamberti \& Martelli (1971) and Lamberti \& Bleve-Zacheo (1979).

In our studies, $X$. pachtaicum was found associated with several plant species. Of these, cherry and sour cherry were already reported (Jenser, 1985), while black locust, European black pine and maple are new host records for Hungary.

This species is widespread in Europe and it has been shown to occur in several countries of Central and Eastern Europe, such as Bulgaria (Choleva, 1975, Lamberti et al., 1983), Croatia (Samota et al., 1994), the Czech Republic (Kumari, 2004, Kumari et al., 2005), Macedonia, Montenegro and Serbia (Barsi \& Lamberti, 2002), Moldavia and Ukraine (Brown et al., 1990) and Slovakia (Liskova, 1992, Lamberti et al., 1999).

\section{Xiphinema simile}

Xiphinema simile found in the rhizosphere of maple and grapevine at Gödöllő and at an unknown locality respectively, are morphometrically similar.

The morphometrical characters of these isolates are consistent with the paratypes (Lamberti et al., 1983), except in having longer body (2.3 vs $1.9 \mathrm{~mm})$, higher values for all the ratios related to the body length and longer distance from anterior extremity to guiding ring (60.2 and 58 vs $51 \mu \mathrm{m})$.

The Hungarian isolates closely agree in measurements with populations from Bulgaria (Peneva \& Choleva, 1992), Slovakia (Liskova \& Brown, 1996; Lamberti et al., 1999), former Yugoslavia (Barsi, 1994; Barsi \& Lamberti, 2002, 2004), Czech Republic (Kumari, 2006) and Kenya (Coomans \& Heyns, 1997), except for body length. However, mean body length of Hungarian isolates is similar to those of the Czech and Slovakian but longer than those of the Bulgarian and Kenian populations.

Data on the geographical distribution and associated hosts of Xiphinema simile are available from: Bajsa-Lipar and Zobnatica - grasses and hawthorn (Crataegus monogyna) (Barsi \& Lamberti, 2002); Bosnia and Herzegovina - dogwood (Cornus sp.) and maize (Zea mays) (Barsi \& Lamberti, 2004); Bulgaria - apple, apricot, black currant (Ribes nigrum), grapevine, quince, red oak (Quercus rubra), 
white birch (Betula alba), and white poplar (Populus alba) (Lamberti et al., 1983), Peneva \& Choleva, 1992); Macedonia - sycamore; Serbia and Montenegro - Carduus nutans, Euphorbia cyparissias, Euphorbia sp., grapevine, Pteridium aquilinum, saliferous grassland, and Trifolium campestre (Barsi, 1994, 1996); Serbia (Neradin - Fruska Gora Mountain) - dog-rose (Rosa canina) (Barsi \& Lamberti, 2002); Slovakia - grapevine, horse-chestnut (Aesculus hippocastanum), plum, poplar (Populus sp.), and rose (Rosa sp.) (Lamberti et al., 1983, 1999, Liskova et. al., 1993); the Czech Republic - apple, apricot, sour cherry, and sweet cherry (Kumari, 2006). Outside Europe, until now this species has been found only in Kenya (Coomans \& Heyns, 1997) in Gazi (along the Mara river, near Governo's Camp), on old baobab (Adansonia digitata) and white pepper tree (Warburgia salutaris).

Regarding plant-nematode associations, our results confirm the data from the literature in terms of occurrence on grapevine, cherry, apricot and plum. However, almond, quince, maple and birch are new plant association records for this species.

During the present survey, species of the $X$. americanumgroup were not frequent. Therefore conclusions regarding their host plant preferences cannot be drawn. However, in terms of geographic distribution, these species appear to be spread across the country.

\section{Acknowledgements}

Mutual visits of authors have been supported by a mobility program between Hungarian Academy of Sciences (H.A.S.) and Consiglio Nazionale delle Ricerca (C.N.R.). Part of sampling work was carried out in the framework of OTKA T 48434 grant. Hungarian authors wish to express their appreciation to Professors Gábor Jenser and Gábor Bakonyi for their tutorship and advice. Efforts from unknown reviewers are appreciated since their comments considerably improved the manuscript.

\section{References}

ANDRÁSSY, I. (1979): [Another 30 nematode species in the Hungarian fauna]. Állattani Közlemények, 66: 213-216 ANDRÁSSY, I. (1990): [Free-living nematoda in the Hungarian fauna]. Állattani Közlemények, 57: 17-38

BARSI, L. (1994): Species of the $X$. americanum-group (Nematoda, Dorylaimida) on the territory of the former Jugoslavia. Nematol. Medit., 22: 25-34

BARSI, L. (1996): Occurrence of Xiphinema species in the former Yugoslavia - Supplement to the "Atlas of plant parasitic nematodes of Yugoslavia". Nematol. Medit., 24: 195-199

BARSI, L., LAMBERTI, F. (2002): Morphometrics of three putative species of the Xiphinema americanum group (Nematoda: Dorylaimida) from the territory of the former Yugoslavia. Nematol. Medit., 30: 59-72

BARSI, L., LAMBERTI, F. (2004): Xiphinema parasimile sp.n. Serbia and $X$. simile, first record from Bosnia and Herzegovina (Nematoda, Dorylaimida). Nematol. Medit., 32: 101-109

Brown, D. J. F., TAYlor, C. E., ChOleva, B., ROMANENKO, N. D. (1990): The occurrence of Longidoridae (Nematoda: Dorylaimida) in Western USSR with further comments on longidorid nematodes in Europe and the Mediterranean basin. Nematol. Medit., 18: 199-207

Choleva, B. (1975): Nematodes of the family Longidoridae in Bulgaria. In LAMBERTI, F., TAYLOR, C. E., SEINHORST, J. W. (Eds.): Nematode vectors of plant viruses (pp. 355-356) Plenum Press, London \& New York

CoOmans, A., Heyns, J. (1997): Three species of the Xiphinema americanum-group (Nematoda: Longidoridae) from Kenya. Nematologica, 43: 259-274

De Giorgi, C., De Luca, F., Veronico, P., Cortese, M. R., Di Vito, M., LAMBerti, F. (1999): Application of molecular biology in plant nematology. Helminthologia, 36: 171-173

ERBENOVÁ, M. (1975): Ectoparasitic eelworms of the Xiphinema Cobb in the orchards of the C.S.R. Sb. UVTIZahradnictví (Praha), 2: 79-86

ELEKES, A., VALYI, I. (1980): [A nematological survey on grapevine.] Növényvédelem, 16: 49-57

FleGG, J. J. M. (1967): Extraction of Xiphinema and Longidorus species from soil by a modification of Cobb's decanting and sieving technique. Ann. Appl. Biol. 60: 420437

JENSER, G. (1985): [Xiphinema species in Hungarian orchards] Növényvédelem, 21: 289-292

KUMARI, S. (2004): The occurence of Xiphinema vuittenezi, Xiphinema pachtaicum and Longidorus leptocephalus (Nematoda: Dorylaimida) in the Central Czech Republic. Helmintologia, 41: 103-108

Kumari, S., PolÁK, J., ChoutKA, R. (2005): Plantparasitic nematodes of the genus Xiphinema (Nematoda: Longidoridae) in the vineyards of the Czech Republik. Nematology, 7: 81-93

KUMARI, S. (2006): Xiphinema simile (Nematoda: Longidoridae) in Czech Republik and a note on other Xiphinema species. Helminthologia, 43: 43-50

LAmberti, F., Bleve-ZacheO, T. (1979): Studies on Xiphinema americanum sensu lato with description of fifteen new species (Nematoda: Longidoridae). Nematol. Medit., 7: 51-106

LAMBerti, F., MARTelli, G. P. (1971): Notes on Xiphinema mediterraneum (Nematoda: Longidoridae). Nematologica, 17: 75-81

Lamberti, F., Choleva, B., Agostinelli, A. (1983): Longidoridae from Bulgaria (Nematoda, Dorylaimida) with description of three new species of Longidorus and two new species of Xiphinema. Nematol. Medit., 11: 49-72 Lamberti, F., Ciancio, A., Agostinelli, A., Coiro, M. I. (1991): Relationship between Xiphinema brevicolle and $X$. diffusum with a redescription of $X$. brevicolle and descriptions of three new species of Xiphinema (Nematoda: Dorylaimida). Nematol. Medit., 19: 311-326

Lamberti, F., Sabova, M., De Luca, F., Molinari, S., 
Agostinelli, A., Coiro, M. I., VAlockA, B. (1999): Phenotypic variations and genetic characterization of Xiphinema populations from Slovakia (Nematoda: Dorylaimida). Nematol. Medit., 27: 261-275

LAMBerti, F., Molinari, S., Moens, M., Brown, D. J. F. (2000): The Xiphinema americanum-group. I. Putative species, their geographical occurrence and distribution, and regional polytomous identification keys for the group. Rus. J. Nematol., 8: 65-84

Lamberti, F., Hockland, S., Agostinelli, A., Moens, M., Brown, D. J. F. (2004): The Xiphinema americanumgroup. III. Keys to species identification. Nemat. Medit., 32: $53-56$

Lazarova, S. S., Malloch, G., Oliveira, C. M. G., Hubschen, NeIlson, R. (2006): Ribosomal and mitochondrial DNA analyses of Xiphinema americanumgroup populations. Journal of Nematology, 38(4): 404-410 LIŠKOVÁ, M. (1992): Nematodes- virus vectors in the rhizosphere of tree species in Slovakia. Int. Symposium at the occasion of the 100th Anniversary of the Arboretum Mlynany foundation 1892-1992., pp. 231-236

LiŠKOVÁ, M., BROWN, D. J. F. (1996): Taxonomic validity and ecological relations of Xiphinema pachtaicum and $X$. simile (Nematoda: Dorylamida), two members of the $X$. americanum group occurring in Slovakia. Helminthologia, 33: $137-142$

LiŠKovÁ, M., LAMBERTI, F., SABOVÁ, M., VAlockÁ, B., Agostinelli, A. (1993): First record of some species of Longidorid nematodes from Slovakia. Nematol. Medit., 21: 49-53

LOOF, P. A. A., LUC M. (1990): A revised polytomous key for the identification of species of the genus Xiphinema Cobb, 1913 (Nematoda: Longidoridae) with exclusion of the X. americanum-group). Systematic Parasitology 16: 35-66

Luc, M., Coomans, A., Loof, A. A., Baujard, P. (1998): The Xiphinema americanum-group (Nematoda: Longidoridae). 2. Observations on Xiphinema brevicollum Lor- dello \& da Costa, 1961 and comments on the group. Fund. Appl. Nematol., 21: 475-490

Martelli, G. P., LAmBerti, F. (1967): Le specie di Xiphinema Cobb, 1913 trovate in Italia e commenti sulla presenza di Xiphinema americanum Cobb (Nematoda, Dorylaimoidea). Phytopathologia Mediterranea, 6: 65-85 Molinari, S., De LuCA, F., LAMBerTi, F., De Giorgi, C. (1997): Molecular methods for the identification of longidorid nematodes. Nematol. Medit., 25: 55-61

Molinari, S., LAMBERTi, F., DunCAN, L. W., HALBRENDT, J., Kotcon, J., AbAwi, G. S., Robbins, R. T., NyczePir, A. P., Mchenry, M., Magunacelaya, J. C., Crozzoli, R., Lemos, R. M., Nagy, P., Moens, M., Brown, D. J. F. (2004): SOD polymorphism in Xiphinema americanumgroup (Nematoda: Longidoridae). Nematology, 6: 867-876 NAGY, P. (1999): [First record of two virus-transmitting nematode species, Longidorus attenuatus and Xiphinema italiae]. Növényvédelem, 35: 15

Oliveira C. M. G., Fenton B., Malloch G., Brown D. J. F., NeILSON R. (2005): Development of species-specific primers for the ectoparasitic nematode species Xiphinema brevicolle, $X$. diffusum, $X$. elongatum, $X$. ifacolum and $X$. longicaudatum (Nematoda: Longidoridae) based on ribosomal DNA sequences. Annals of Applied Biology, 146: 281-288

Peneva, V., Choleva, B. (1992): Nematodes of the family Longidoridae from forest nurseries in Bulgaria. II. The genus Xiphinema Cobb, 1913. Helminthology, 32: 4658

Repasi, V., Nagy, P., Coiro, M. I., Agostinelli, A., LAMBERTI, F. (2006): [Novel data on the distribution of dagger nematodes in Hungary.] Növényvédelem, 42: 655661

SAmotA, D., IVEZIC, M., RASPUDIC, E. (1994): Ecology of Xiphinema vuittenezi and Xiphinema pachtaicum in vineyards of north-east Croatia. Bulletin OEPP/EPPO Bulletin, 24: 375-381

ACCEPTED APRIL 4, 2008 